\title{
aumento da má conduta científica nas pesquisas da área de saúde: impressão ou realidade?
}

\author{
The increase in scientific misconduct in \\ health research: impression or reality?
}

\section{Diego Ribeiro Rabelo' Gustavo Ferreira Lopes ${ }^{2}$ (1) Henrique Santana Cumming 3 (1)}

\footnotetext{
${ }^{1}$ Autor para correspondência. Escola Bahiana de Medicina e Saúde Pública (Salvador). Bahia, Brasil. diegorabelo@bahiana.edu.br ${ }^{2-3}$ Escola Bahiana de Medicina e Saúde Pública (Salvador). Bahia, Brasil. gustavolopes18.1@bahiana.edu.br, henriquecumming18.1@bahiana.edu.br
}

Lembra quando você descobriu que não existe um bom velhinho que gosta de se vestir de vermelho e todos os anos sai do Polo Norte e viaja o mundo entregando presentes para as crianças comportadas? Ao sermos enganados, nossa confiança no próximo se reduz em vigência do medo de experimentar o sabor da frustração novamente e a tendência é que nos tornemos mais críticos quanto às informações advindas de terceiros. ${ }^{1}$ Portanto, a confiança é essencial para a solidificação das relações humanas ${ }^{2}$ e quando aquela é quebrada essas são fragilizadas.

Recentemente o ecossistema científico despertou para o gosto amargo de ser enganado, quando se deu conta de que a quantidade de má conduta científica na área de saúde é alta. ${ }^{3,4}$ Má conduta científica diz respeito a "qualquer ação que envolva tratamento inadequado dos sujeitos envolvidos na pesquisa ou manipulação proposital dos registros científicos de tal forma que não reflitam a verdade". $\underline{5}$ Ela pode se materializar de diversas formas: falsificação e fabricação de dados, plágio, publicação duplicada e revisão por pares comprometida. ${ }^{3,6-8}$
Porém, não é de hoje que as más condutas científicas existem. Em 1912, aconteceu um dos mais famosos casos de fraude científica relatados, a emblemática história do "homem de Piltdown". Se não fosse o arqueólogo E. T. Hall, que desenvolveu os métodos científicos capazes de escancarar o fato, é possível que até hoje estivéssemos acreditando ter encontrado o "elo perdido". ${ }^{9}$ Avançando no tempo, esse tipo de prática ganhou destaque novamente quando se tornou um problema nos Estados Unidos nas décadas de 70/80. Nessa ocasião, numerosos casos de pesquisas fraudulentas foram descobertos em instituições de prestígio acadêmico, revelando um provável comprometimento de diversas informações disponíveis, antes consideradas "de alta qualidade". $\underline{.}$

Existem outros casos paradigmáticos e lembraremos aqui de dois recentes e igualmente importantes: o do sul-coreano Hwang Woo-Suk que em 2004 declarou ter conseguido, via clonagem, produzir embriões humanos e imediatamente no ano seguinte foi desmascarado $\frac{10}{}$; e o da conspiração da indústria do açúcar, em 1967, em que cientistas americanos foram pagos para fabricar os 
resultados de suas pesquisas, favorecendo o aumento do risco de doenças cardiovasculares por gordura saturada, situação descoberta apenas em 2016.11

\section{O combate à má conduta científica}

Uma vez que, em um contexto científico, sentimos que fomos enganados, assim como no caso do Papai Noel, passamos a tentar nos proteger de outras mentiras e consequentemente aumentamos a criticidade não só com os estudos publicados, mas também com o processo de produção das evidências científicas. ${ }^{12}$ Na prática, foi exatamente isso que aconteceu: a ciência passou a ser mais vigiada e cobrada em relação a práticas de transparência. Partimos de modelos científicos marcados por mecanismos de tentativa-erro e evoluímos para, dentre outros, os atuais processos de revisão por pares e detecção de plágio através de softwares. $\frac{13,14}{14}$ Tais abordagens possuem papel fundamental na melhoria do atual ecossistema científico, reverberando em mais transparência e em um ambiente de menos más práticas. ${ }^{15}$ Atualmente, os números de má conduta reportados estão maiores do que em décadas passadas. Tendo como base a remoção da literatura dos artigos pelas revistas onde foram publicados, as taxas de má conduta entre os anos de 1960-2000 não passavam de 10-27\% dos artigos analisados, enquanto que atualmente, esta marca passa dos 40\%. ${ }^{3}$ Quando situações assim são identificadas, podem ser adotados diversos posicionamentos como a publicação de um artigo com correções, indicando os erros do trabalho original ou, em outras situações, a própria revista pode publicar uma nota expressando sua preocupação quanto à confiabilidade das informações divulgadas em um determinado artigo. ${ }^{16}$ Inclusive, o número de revistas que reportam retratações tem aumentado ao longo dos anos: em 1997 eram apenas 44, enquanto que em 2016 este número aumentou para 488.17

Nesse sentido, como não poderia seria diferente, ao tentar reproduzir as informações geradas por um modelo de produção com tais características, muitas vezes não são encontrados os mesmos resultados, o que em nada contribui com a evolução do conhecimento científico. ${ }^{18}$ Além disso, um forte motivo para levar à retratação de estudos é a utilização de informações falsas ou manipuladas, os seus resultados podem não ser aplicáveis na prática. $\frac{19}{} \mathrm{E}$ assim, por consequência, a busca por evidências científicas de qualidade se torna um trabalho complexo, o que pode comprometer a atuação de profissionais da saúde que utilizam essas informações para tomar decisões clínicas. $\frac{15}{5}$

Como resposta, diversas iniciativas pelo mundo buscaram entender e corrigir distorções do ecossistema científico ao longo dos anos. Em 1978, foi criado o International Committee of Medical Journal Editors ICMJE, um pequeno grupo de trabalho formado por editores de revistas científicas de medicina generalista que se encontram anualmente para discutir sobre recomendações de conduta, relato, edição e publicação de trabalhos acadêmicos. 20 Já em 1997, a primeira reunião do Committee on Publication Ethics - COPE aconteceu, sendo essa, mais tarde, uma das mais relevantes iniciativas na criação de guidelines de retratação para área de saúde. ${ }^{21} \mathrm{Em}$ 2012, um grupo de editores de periódicos se reuniu para criação da Declaração de São Francisco sobre Avaliação da Pesquisa, no intuito de afirmar que há uma necessidade de aprimorar a maneira que as agências de financiamento, instituições acadêmicas e outros grupos avaliam a produção da pesquisa científica ${ }^{22}$, visto que o sistema de incentivos e as recompensas para publicar são corresponsáveis pela má prática em pesquisa ${ }^{6,23-25}$ ao fomentarem, por exemplo, revisões por pares falsas e autorias inapropriadas. ${ }^{26}$

Três anos depois, em 2015, o Manifesto de Leiden coloca em pauta a importância do juízo de valor dos especialistas como um complemento às avaliações de conteúdo dos artigos, não reduzindo esse processo à avaliação exclusiva por métricas. 27,28 Nesse mesmo ano, o projeto COMPare checou de forma sistemática todos os ensaios publicados em 5 revistas médicas de boa reputação, analisando se estes periódicos relataram incorretamente suas descobertas. $\underline{29} \mathrm{Em}$ 2018, o The People's Trial começou uma iniciativa buscando imergir a população comum na comunidade científica através do incentivo à busca de respostas para questionamentos antes tidos como verdades absolutas. ${ }^{30}$ Criada também em 2018, a Iniciativa Brasileira de Reprodutibilidade se configura como um grupo que, motivados pelos indícios de baixa validade interna das publicações científicas e a forte cultura do publique ou pereça, busca quantificar a reprodutibilidade da ciência biomédica brasileira e portanto, quanto dos seus resultados são confiáveis. ${ }^{31}$,32 Já no ano de 2019, a produção científica de má qualidade foi pauta da $6^{\mathrm{a}}$ Conferência Mundial sobre Integridade Científica em Hong Kong, encontro 
no qual foram levantados diversos questionamentos acerca do comportamento dos pesquisadores, no intuito de repensar a forma como a produção científica é recompensada. $\underline{33}$

A existência desses órgãos em si não indica a magnitude e a extensão das más práticas científicas na área biomédica, mas alerta para um problema grave e com potenciais impactos devastadores. Todavia, é possível afirmar que há uma crise estabelecida ${ }^{34}$ ? A disponibilidade de material científico na internet foi um marco para essa desconfiança, visto que ao mesmo tempo em que permitiu que mais dados fossem manipulados de forma indevida, também favoreceu a criação de novas iniciativas e tecnologias para detectar algumas infrações de forma mais rápida e precisa. $\frac{35}{5}$

Entretanto, a palavra crise pressupõe que atualmente há mais má conduta científica do que antes, mas será que estamos sendo vítimas de mais falcatruas do que no passado ou apenas nos tornamos mais críticos para com as informações publicadas e passamos a perceber com maior precisão quando estamos consumindo informações cientificamente questionáveis?

\section{Impressão ou realidade?}

Esse dilema poderia ser resolvido pela própria (meta) ciência. Todavia, a maior parte dos artigos que argumentam a favor de uma crise de má conduta científica são descritivos, restringem-se às especulações. Dentre os poucos trabalhos originais encontrados que testaram empiricamente tal hipótese consta o estudo de Cokol M. realizado em 2008. Nele, comparou-se o número de publicações com o percentual de artigos retratados ao longo dos anos, evidenciando que entre 1980 e 2000 a proporção de retratações não foi constante. ${ }^{4}$ Já em 2011, R Grant Steen investigou o número e a justificativa para a retratação dos artigos publicados na língua inglesa entre 2000 e 2010, revelando um salto de 40 retratações no ano de 2003 para 180 no ano de 2009, sendo os principais motivos erro científico, plágio, fabricação e falsificação de dados. ${ }^{3}$ Além disso, em 2013, R Grant Steen publicou outro trabalho avaliando o tempo entre a publicação de um artigo e a sua retratação, mostrando que o tempo médio para a publicação de uma retratação era de 49,82 meses antes de 2002, enquanto que após o ano de 2002 esse tempo foi reduzido para
23,82 meses, sugerindo que as revistas científicas estão retratando os artigos mais rapidamente. $\frac{36}{6}$

Portanto, há sinais de que há um aumento e aceleração na detecção do número de más condutas científicas por ano. Por outro lado, é inegável o fato de que há um maior esforço por parte da comunidade científica em avaliar a literatura publicada através de iniciativas, recursos otimizados e buscas melhoradas de artigos defeituosos. Logo, apesar dos indícios, não fomos capazes de encontrar evidências científicas suficientes para afirmar que a má conduta em ciência tem aumentado independentemente de outros fatores, podendo isto ser contribuição de uma fiscalização maior do ecossistema científico, da sofisticação dos conceitos de práticas científicas questionáveis ou até mesmos de todos estes fenômenos se desenvolvendo concomitantemente.

Enquanto essas evidências não aparecem, como qualquer cientista que se preze, não podemos descartar a hipótese de que há uma crise de má conduta científica estabelecida. Porém, pensamos que, como sociedade, precisamos refletir e produzir mais dados sobre esse tema, pois provavelmente a descoberta da fraude do Papai Noel não trouxe muitos prejuízos às nossas vidas, mas no caso da referida crise ser real, vidas estão em risco.

\section{Contribuições dos autores}

Todos os autores participaram de todas as etapas da concepção à revisão final do manuscrito.

\section{Conflitos de interesses}

Nenhum conflito financeiro, legal ou político envolvendo terceiros (governo, empresas e fundações privadas, etc.) foi declarado para nenhum aspecto do trabalho submetido (incluindo, mas não se limitando a subvenções e financiamentos, participação em conselho consultivo, desenho de estudo, preparação de manuscrito, análise estatística, etc.).

\section{Referências}

\footnotetext{
1. Mazar N, Amir O, Ariely D. The dishonesty of honest people. J Mark Res. 2008;45(6):633-44. https://doi. org/10.1509\%2Fjmkr.45.6.633
} 
2. Vlaar PWL, Van Den Bosch FAJ, Volberda HW. On the evolution of trust, distrust, and formal coordination and control in interorganizational relationships: Toward an integrative framework. SAGE Open. 2007;32(4):407-28. https://doi. org/10.1177\%2F1059601106294215

3. Steen RG. Retractions in the scientific literature: Is the incidence of research fraud increasing? J Med Ethics. 2011;37(4):249-53. https://doi.org/10.1136/jme.2010.040923

4. Cokol M, Ozbay F, Rodriguez-Esteban R. Retraction rates are on the rise. EMBO Rep. 2008;9(1):2. https://doi.org/10.1038/ sj.embor. 7401143

5. Rode SM, Rios R, Oliveira F, Paranhos LR. Má conduta em publicações científicas. 2018;23(3):7-8. https://doi. org/10.1590/2177-6709.23.3.007-008.edt

6. Fang FC, Steen RG, Casadevall A. Misconduct accounts for the majority of retracted scientific publications. Proc Natl Acad Sci U S A. 2012;109(42):17028-33. https://doi.org/10.1073/ pnas.1212247109

7. Almeida RMVR, Rocha KA, Catelani F, Fontes-Pereira AJ, Vasconcelos SMR. Plagiarism Allegations Account for Most Retractions in Major Latin American/Caribbean Databases. Sci Eng Ethics. 2016;22(5):1447-56. https://doi.org/10.1007/s11948-0159714-5

8. Moylan EC, Kowalczuk MK. Why articles are retracted: A retrospective cross-sectional study of retraction notices at BioMed Central. BMJ Open. 2016;6(11):e012047. http://dx.doi.org/10.1136/ bmjopen-2016-012047

9. Yawar A. For the love of Piltdown Man. Lancet Neurol. 2018;17(7):586. https://doi.org/10.1016/s1474-4422(16)30309-x

10. Cyranoski D. Woo Suk Hwang convicted, but not of fraud. Nature. 2009;461(7268):1181. https://doi.org/10.1038/4611181a

11. Kearns CE, Schmidt LA, Glantz SA. Sugar industry and coronary heart disease research: $A$ historical analysis of internal industry documents. JAMA Intern Med. 2016;176(11):1680-5. https://doi. org/10.1038/4611181a

12. Mazar N, Ariely D. Dishonesty in scientific research. J Clin Invest. 2015;125(11):3993-6. https://doi.org/10.1172/jci84722

13. David PA, Spence MJ. Towards Institutional Infrastructures for e-Science: The Scope of the Challenge. SSRN Electron J. 2011;(2):198. http://dx.doi.org/10.2139/ssrn.1325240

14. Targino MG, Garcia JCR, Silva KLN. Information science reviewers versus the open peer review. Rev Interam Bibliotecol. 2019;43(1):e5. https://doi.org/10.17533/udea.rib.v43n1ei3

15. Murphy SP, Bulman C, Shariati B, Hausmann L. Submitting a manuscript for peer review-integrity, integrity, integrity. J Neurochem. 2014;128(3):341-3. https://doi.org/10.1111/jnc.12644
16. Bauchner H, Fontanarosa PB, Flanagin A, Thornton J. Scientific misconduct and medical journals. JAMA. 2018;320(19):1985-7. https://doi.org/10.1001/jama.2018.14350

17. Brainard J, Jia Y. What a massive database of retracted papers reveals about science publishing's 'death penalty' [Internet]. Science Mag; 2018 [citado em 2021 Mar 5]. Disponível em: https:// www.sciencemag.org/news/2018/10/what-massive-databaseretracted-papers-reveals-about-science-publishing-s-deathpenalty

18. Ioannidis JPA. Why Most Clinical Research Is Not Useful. PLoS Med. 2016;13(6):e1002049. https://doi.org/10.1371/journal. pmed.1002049

19. Mesquita CT. Integrity in Scientific Research. Int J Cardiovasc Sci. 2017;2(1):1-2. Disponível em: https://doi.org/10.5935/23594802.20170026

20. ICMJE [Internet]. International committee of medical journal editors; 2020 [citado em 2020 Out 26]. Disponível em: http://www. icmje.org/about-icmje/

21. Committee on Publication Ethics. COPE history timeline [Internet]. COPE; 2020 [citado em 2020 Nov 26]. Disponível em: https://publicationethics.org/about/history

22. Flaquer GN, Gomes MP, Silveira L, Michels PE, Vidal I. Declaração de São Francisco sobre Avaliação da Pesquisa [Internet]. DORA; 2012. Disponível em: https://sfdora.org/wpcontent/uploads/2018/11/DORA Brazilian-Portuguese.pdf

23. Fang FC, Casadevall A. Reforming science: Structural reforms. Infect Immun. 2012;80(3):897-901. https://dx.doi. org/10.1128\%2FIAI.06184-11

24. Casadevall A, Fang FC. Reforming science: Methodological and cultural reforms. Infect Immun. 2012;80(3):891-6. https://doi. org/10.1128/iai.06183-11

25. Palla IA, Singson $M$, Thiyagarajan S. A comparative analysis of retracted papers in Health Sciences from China and India. Account Res. 2020;27(7):401-16. https://doi.org/10.1080/08989621.2020.1 $\underline{754804}$

26. Rivera H. Fake peer review and inappropriate authorship are real evils. J Korean Med Sci. 2019;34(2):e6. https://dx.doi. org/10.3346\%2Fjkms.2019.34.e6

27. Hicks D, Wouters $P$, Waltman L, Rijcke S, Rafols I. The Leiden Manifesto for research metrics. Nature. 2015;520(7548):429-31. https://doi.org/10.1038/520429a

28. Benedictus R, Miedema F, Ferguson MWJ. Fewer numbers, better science. Nature. 2016;538(7626):453-5. https://doi. org/10.1038/538453a 
29. Goldacre B, Drysdale H, Powell-Smith A, Dale A, Milosevic I, Slade $E$, et al. The compare Trials Project [Internet]. Oxford: Centre for Evidence-Based Medicine; 2016. Disponível em: www. compare-trials.org

30. The People's Trial [Internet]. NUI Galway, Irlanda: Health Research Board; 2019 [citado em 2020 Out 26]. Disponível em: https://thepeoplestrial.ie/about-us/

31. Iniciativa Brasileira de Reprodutibilidade [Internet]. Rio de Janeiro: Instituto de Bioquímica Médica Leopoldo de Meis / Universidade Federal do Rio de Janeiro / Instituto Serrapilheira. 2020 [cited 2020 Oct 26]. Available from: https://www.

reprodutibilidade.bio.br/

32. Neves K, Carneiro CFD, Wasilewska-Sampaio AP, Abreu M, Valério-Gomes $\mathrm{B}$, Tan PB, et al. Two years into the brazilian reproducibility initiative: Reflections on conducting a large-scale replication of brazilian biomedical science. Mem Inst Oswaldo Cruz. 2020;115(9):e200328. https://doi.org/10.1590/007402760200328
33. Moher D, Bouter L, Kleinert S, Glasziou P, Sham MH, Barbour $\checkmark$, et al. The Hong Kong Principles for assessing researchers: Fostering research integrity. PLoS Biol. 2020;18(7):e3000737. https://doi.org/10.1371/journal.pbio.3000737

34. Twa MD. Scientific Integrity and the Reproducibility Crisis. Optom Vis Sci. 2019;96(1):1-2. https://doi.org/10.1097/ opx.0000000000001339

35. Long TC, Errami M, George AC, Sun Z, Garner HR. Scientific intergrity: Responding to possible plagiarism. Science.

2009;323(5919):1293-4. https://doi.org/10.1126/science.1167408

36. Steen RG, Casadevall A, Fang FC. Why Has the Number of Scientific Retractions Increased? PLoS One. 2013;8(7):e68397. https://dx.doi.org/10.1371\%2Fjournal.pone.0068397 